\title{
Design and Research of Physical Education Platform Based on Artificial Intelligence
}

\author{
Yufeng Ba $\mathbb{D D}^{1}$ and Zhenfeng Liu $\mathbb{D D}^{2}$ \\ ${ }^{1}$ Institute of Physical Education, Henan Agriculture University, Zhengzhou 450046, Henan, China \\ ${ }^{2}$ School of Physical Education, Henan University, Kaifeng 475001, Henan, China \\ Correspondence should be addressed to Zhenfeng Liu; lzfgkx@163.com
}

Received 25 November 2021; Revised 25 December 2021; Accepted 12 January 2022; Published 22 February 2022

Academic Editor: Le Sun

Copyright (c) 2022 Yufeng Ba and Zhenfeng Liu. This is an open access article distributed under the Creative Commons Attribution License, which permits unrestricted use, distribution, and reproduction in any medium, provided the original work is properly cited.

With the continuous development of information technology, conventional physical education teaching methods are no longer applicable. In order to ensure the objectivity of college physical education evaluation, this paper designs a set of college physical education management information system based on artificial intelligence technology. The weighting algorithm in the student performance evaluation module and teacher performance evaluation module in the system adopts the intelligent algorithm based on FNN neural network. Experimental verification shows that the intelligent algorithm based on the FNN neural network can effectively predict the students' score in the national college physical education examination, which can provide a more objective basis for teacher performance evaluation.

\section{Introduction}

Wang Yuqing said in the study that physical education in colleges and universities in China is divided into physical education major, physical education major, and nonphysical education major [1]. Wang Ming said in the study which professional terms such as sports management and competition management are nonphysical education majors [2]. Li Feifei said that, according to the requirements of the Ministry of Education, nonsports majors need to receive at least 2 hours of formal physical education every week [3]. Wen Jiao said in a study on college physical education that the premise for students to obtain graduation qualification is that their physical performance passes the national unified physical education examination [4]. Li Zhang has proposed the university sports test content in the study. The test contents include middle and long distance running $(1500 \mathrm{~m}$ for boys and $800 \mathrm{~m}$ for girls), standing long jump or high jump, throwing (shot put, rubber ball, and long handle grenade throw are optional), physical flexibility (generally sitting forward flexion), and learning a competition [5]. LiuYanRu also further explained to the college sports test that, in addition to competition events, running, jumping, throwing, flexibility, and other tests all pass the score line strictly [6]. Zhang Jianye explains the other events in the college sports tests: competition events can generally be selected from badminton, tennis, table tennis, basketball, volleyball and other ball games, or martial arts [7].

Li Zihao carried out research on the effectiveness of physical education teaching in universities. In the early research, students' physical education achievements were only constrained by the unified examination of national college physical education curriculum, so too many examination oriented elements were integrated into physical education [8]. The study believes that the core goal of college physical education should be to improve students' comprehensive physical quality and ensure students' physical and mental health during their study in school. Li Xianshu considers that students' physical and mental health is difficult to be effectively controlled by the traditional index factor method [9], so artificial intelligence algorithm is introduced to construct fuzzy evaluation factors for students' physical and mental health, so as to evaluate the achievements of physical education in colleges and universities. 
Fuzzy factor method uses fuzzy neural network (FNN) to analyze multiple controllable indexes of students. Gaobin illustrates the FNN, indicating that FNN is a double variable that presses the data into a $[0,1]$ interval $[10]$ and uses this variable to control the teaching results of physical education teachers.

\section{Comprehensive Evaluation Model of College Students' Physical Education Achievements}

Yang Dapeng in his study proposed that, in the actual management of college students, students' physical fitness test and physical education examination can directly reflect students' physical education curriculum level [11]. YongBai (2016) stated in the study that, in addition, students' vital capacity, body fat rate, and other indicators were investigated by general surgical examination in students' regular physical examination and students' SAS and SDS level in general psychiatric examination can reflect students' physical and mental health from a certain angle [12]. The logical relationship between the above four categories of data and related subcategories is shown in Figure 1.

In Figure 1, the general surgical examination data and physical examination data in physical examination belong to dimensional data, which can be recognized by FNN only after dimensionless normalization. Other data belong to dimensionless data and can be directly input into FNN. The fuzzy convolution result of FNN can be directly used as the fuzzy evaluation result of students' physical education achievement. The results of the above dimensionless normalization algorithm is shown (1) as

$$
y_{i}=\frac{x_{i}-\min (x)}{\max (x)-\min (x)},
$$

where $x_{i}$ is the $i$ input value in $x$ sequence, $y_{i}$ is the dimensionless output value corresponding to the $i$ input value in sequence $x, \min (x)$ is the minimum value of sequence $x$, and $\max (x)$ is the maximum value of sequence $x$.

The data sorted by the above minmax module are all data in the $[0,1]$ interval, while mental health data such as SDS and SAS and physical examination result data are dimensionless data. If there are no special requirements, SDS and SAS will count in the $[0,10]$ interval according to the 10 point system, and the physical examination results will count in the [0100] interval according to the 100 point system. In order to reduce the complexity of the statistical process of this module, when it is required to issue SDS and SAS evaluation, directly input the 1-point system results; that is, the original results are formed in the $[0,1]$ interval, and the physical examination results are also directly issued in the 1point system results; that is, the original results are formed in the $[0,1]$ interval.

The statistical significance of FNN neural network is to summarize all the above input data into a double precision data as the actual evaluation result data of students. If there is no subsequent data processing, FNN neural network will be trained to converge to a double precision variable in the $[0,1]$ interval. This variable is multiplied by 100 to form a 100 point evaluation result as the final physical education achievement of students. The node function of the neural network selects polynomial depth iterative regression function (2):

$$
y=\sum_{i=1}^{n} \sum_{j=0}^{5} A_{j} x_{i}^{j},
$$

where $n$ is the number of nodes of the previous neural network, $j$ is the polynomial order, and $A_{j}$ is the coefficient to be regressed of the $j$ order polynomial. Other mathematical symbols have the same meaning as (1).

\section{Construction of Artificial Intelligence System for College Physical Education}

Based on the comprehensive analysis of the above neural network model, it is found that, in addition to the system administrator, the system needs four roles, namely, the assessed students, physical education teachers, surgeons, and psychologists. Surgeons fill in the results of general surgical examinations, psychologists fill in the results of SAS and SDS scales, and physical education teachers fill in the results of the national unified physical education examination and physical fitness test in colleges and universities. In addition to cooperating with the above four inspections, students have the authority to query the transcripts. The transcripts will also be submitted to the Academic Affairs Office for teaching quality evaluation and to the student work office for student performance summary management. The system architecture is shown in Figure 2:

In Figure 2, FNN neural network and predata processing process have been deeply analyzed in the previous text. Here, we focus on the postprocessing of students' transcripts. The system provides the general query function of students' transcripts; that is, entering the student number can query the transcripts of the specified students, and entering the class name can access the summary table of the transcripts of the whole class, including the descending sorting of scores, and the classified statistics of excellent (80-100 points), pass (60-80 points), and fail (0-60 points). At the same time, teachers' postperformance is evaluated according to the change and distribution of students' performance. Because other statistical work algorithms are relatively simple and limited by space, they are not discussed here. Only the teacher postperformance evaluation algorithm is expanded as Figure 3.

In Figure 3, another group of FNN neural networks is used to evaluate teacher performance. The node function of the neural network is consistent with that of the FNN network in Figure 2. Refer to (2), and the data preprocessing algorithm before the neural network refers to (1). If the traditional teacher performance evaluation scheme based on the weighted factor method is adopted, because the weighted factor itself has a systematic error and the systematic error may be aimed at different students' basic physical conditions, differences in colleges and departments, differences in students' gender and age, etc., the evaluation reliability cannot be effectively 


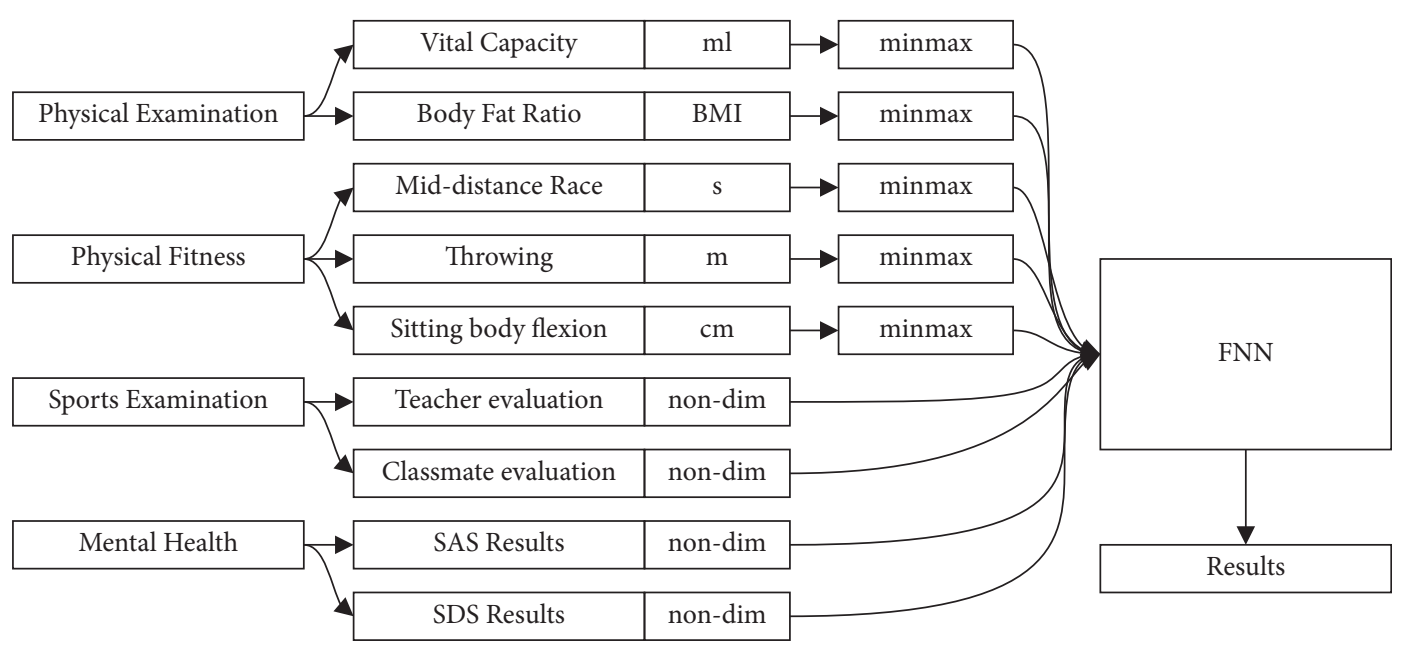

FIGURE 1: Evaluation index of physical education in colleges and universities and its machine learning analysis.

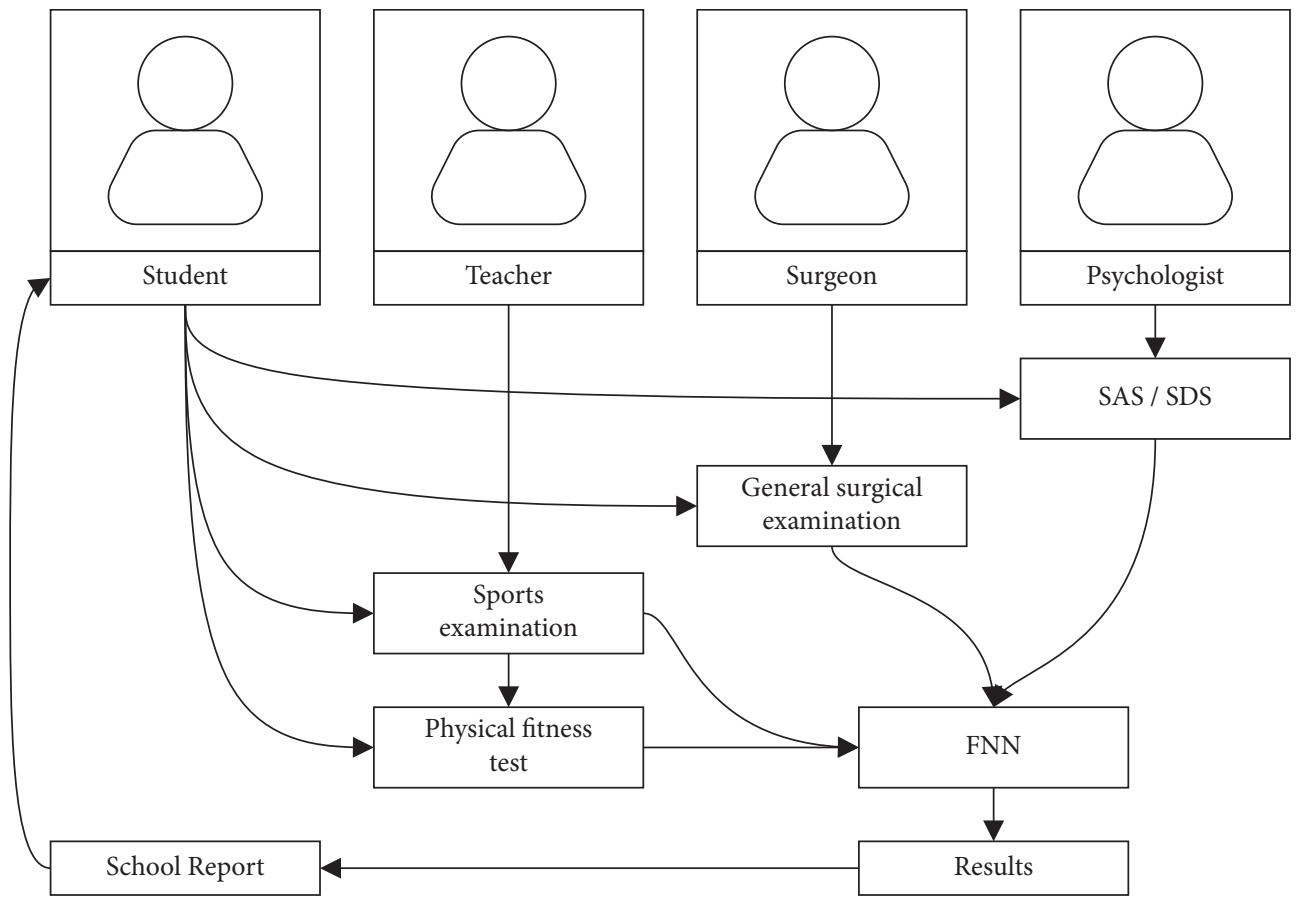

FIgURE 2: Architecture diagram of the artificial intelligence system (FNN) for physical education teaching evaluation in colleges and universities.

guaranteed. In this study, FNN neural network is selected for the comprehensive evaluation of students' physical education performance, and it also realizes the comprehensive evaluation of teachers' performance.

\section{Overall Simulation Verification of System Effectiveness}

Taking the real original files of 21 nonsports majors in 2018 and 2019 in 2020-2021 academic year as the data source, the analysis environment is constructed in MATLAB, the traditional method is used to analyze student performance and teacher performance as the reference group, and the artificial intelligence method designed in this study is used to analyze student performance and teacher performance as the observation group. The following three validation studies were carried out.

4.1. Correlation between Artificial Intelligence Evaluation and National Physical Education Examination Results. In order to verify the role of the artificial intelligence method designed in this study in student achievement and teacher achievement, the R2 value was obtained by the linear regression method under SPSS, and $t$ value and $P$ value were obtained by bivariate $t$ calibration.

The $\mathrm{R} 2$ values were counted as the ratio of the regression residue to the mean residue (3): 


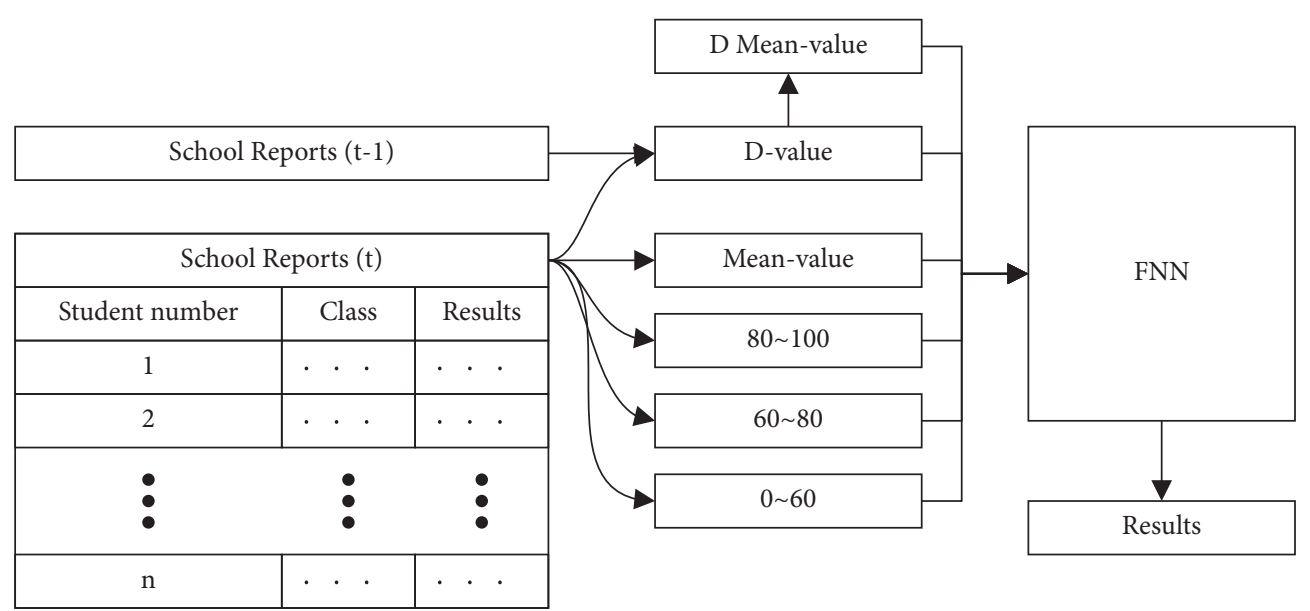

FIGURE 3: Schematic diagram of the teacher performance evaluation system.

$$
\begin{aligned}
R^{2} & =\frac{\sum_{i}\left(x_{i}-\bar{x}\right)}{\sum_{i}\left(x_{i}-\tilde{x}_{i}\right)}, \\
\bar{x} & =\frac{1}{n} \sum_{i=1}^{n} x_{i},
\end{aligned}
$$

where $\bar{x}$ is the mean value of the test sample sequence, $x_{i}$ is the regression value in the sequence, $i$ is the input value in the sequence, and $n$ is the number of test samples.

The $T$ value and $P$ value of bivariate t-check come from the bivariate t-check process, where $t$ value is the value value of the output result. When $t>10.000$, it is considered that there is a statistical difference between the two columns of data, and the greater the $T$ value, the greater the statistical difference; the $P$ value is the log value of the output result. When $p<0.05$, it is considered that the result data are within the confidence space. When $p<0.01$, it is considered that the result data have a significant statistical significance. The smaller the $p$ value, the higher the degree of confidence. Subject to the length, only the calculation algorithm of $T$ value (value) is explained here (4):

$$
\begin{aligned}
t_{\text {Value }} & =\frac{\bar{x}-\mu}{\left(\sigma_{x} / \sqrt{n-1}\right)}, \bar{x}, \\
\mu & =\frac{1}{n, m} \sum_{i=1}^{n, m} x_{i}, \\
\sigma_{x} & =\frac{1}{n-1} \sqrt{\sum_{i=1}^{n}\left(x_{i}-\bar{x}\right)^{2},}
\end{aligned}
$$

where $\mathrm{x}$ means the average value of the investigation sample sequence and $\mu$ means the average value of the reference sequence. $n$ is the number of nodes of the investigation sample sequence, $\mathrm{m}$ is the number of nodes of the reference sample sequence, and $\sigma$ means the standard deviation rate of the investigation sample sequence. Firstly, the linear regression method is used to calculate the correlation between the national unified examination results and the comprehensive evaluation results of the system, and Figure 4 is obtained.

In Figure 4, there is a significant linear relationship between the results of the National Physical Education unified examination and the comprehensive evaluation value of the system, $R^{2}=0.9509$ and $P=0.003$, because the comprehensive evaluation process of the system does not introduce the results of the National Physical Education unified examination, but according to the results of the physical education classroom teaching test, it can be considered that the system has a certain predictive value for the results of the National Physical Education unified examination. The $R^{2}$ value calculation scheme is the ratio of regression variance to linear variance, which can calculate the difference between the regression result and the original result.

In the actual correlation analysis, in addition to the results of the National Physical Education unified examination, it also investigates the results of SDS and SAS evaluation, comprehensive physical examination evaluation, and surgical examination evaluation of students in the evaluation of students' mental health and introduces the bivariate $t$-test analysis results of the above evaluation results and the comprehensive evaluation results of artificial intelligence given by the system. The above analysis results are shown in Table 1 .

In Table 1 , there are significant statistical consistency $(T>10.000, p<0.01)$ and linear correlation $(R 2>0.75$, $p<0.01)$ between the five data and the comprehensive evaluation results of artificial intelligence given in this study. However, there are some statistical differences between the relevant data. For example, the comprehensive evaluation results of artificial intelligence given by the system are highly correlated with the results of the national physical examination, physical fitness test, and general surgical examination (both $T$ value and R2 value are large), but slightly less correlated with the results of mental health examination (SAS and SDS) (both $T$ value and R2 value are small).

In Figure 5, the correlation between the national unified assessment results and the comprehensive evaluation of the artificial intelligence system is calculated by the linear 


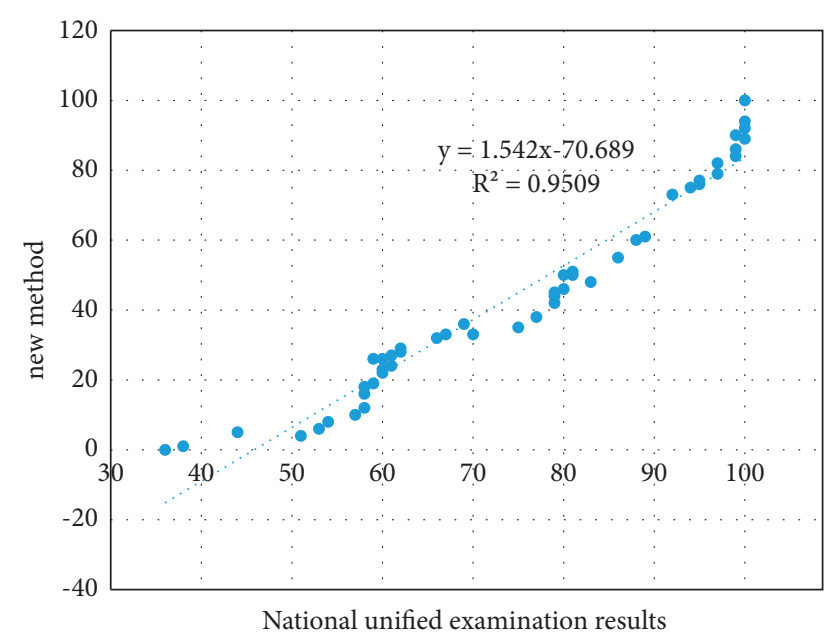

FIGURE 4: Linear regression results of national unified examination results and comprehensive evaluation results of the system.

regression method to compare the differences between the original assessment items and artificial intelligence system.

\subsection{After Using the Artificial Intelligence Comprehensive} Evaluation System, the Statistical Results of Teachers' Performance Change. There are 24 PE teachers in our school. The evaluation results of the original teaching evaluation teachers' performance are counted, and then, the artificial intelligence teacher performance evaluation method given by the system is used to evaluate the teachers' performance. The comparison results are shown in Table 2 .

In Table 2, on the premise that the evaluation scheme remains unchanged, the probability of teachers in the physical education teaching group obtaining advanced teachers decreases from $25 \%$ to $17 \%$. Among the four teachers who obtain advanced teachers in the new scheme, three teachers are different from the previous evaluation result list, accounting for $75 \%$, while the probability of backward teachers increases from $21 \%$ to $33 \%$, and one teacher is different from the previous evaluation result list, accounting for $18 \%$. That is, the new scheme has more stringent requirements for teachers and puts forward higher requirements for college physical education teachers.

In Figure 6, through the analysis of the correlation between the old and new schemes on teachers' performance evaluation results and using the artificial intelligence teachers' performance evaluation method given by the system to evaluate teachers' performance, it is found that the probability of obtaining senior teachers has decreased by $8 \%$, the performance evaluation of teachers is more strict, and the probability of teachers with poor evaluation is higher, and it is concluded that the new scheme has higher requirements for PE teachers and the judgment results are more rigorous.

\subsection{Investigation and Statistics of Teachers' and Students'} Subjective Feelings about the Evaluation Results of the System. Because the above simulation process is aimed at the student performance and teacher performance in the academic year
TABLE 1: Correlation analysis between relevant data and comprehensive evaluation results of the system.

\begin{tabular}{lcccc}
\hline Comparison items & \multicolumn{2}{c}{$\begin{array}{c}\text { Regression } \\
\text { analysis }\end{array}$} & \multicolumn{2}{c}{$\begin{array}{c}\text { Bivariate } \mathrm{t}- \\
\text { check }\end{array}$} \\
& $R^{2}$ & $P$ & $\mathrm{t}$ & $P$ \\
\hline Unified examination results & 0.9509 & 0.003 & 86.273 & 0.004 \\
SDS & 0.8364 & 0.007 & 65.126 & 0.007 \\
SAS & 0.7913 & 0.006 & 71.235 & 0.007 \\
Physical fitness test results & 0.9758 & 0.002 & 92.715 & 0.004 \\
Surgical examination results & 0.8674 & 0.003 & 88.594 & 0.003 \\
\hline
\end{tabular}

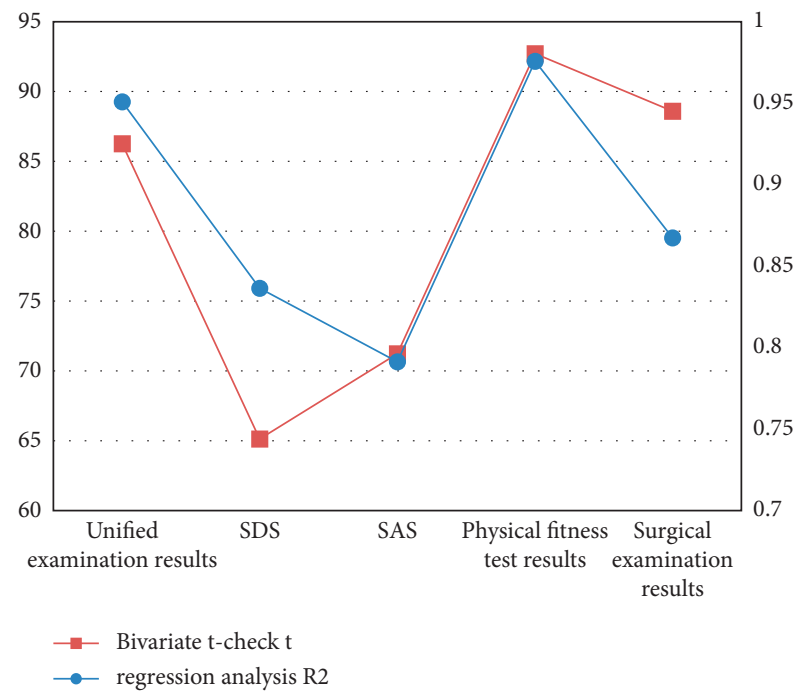

FIGURE 5: Correlation between student assessment items and comprehensive evaluation of artificial intelligence.

2020-2021, 500 students are selected as the survey object, and all 24 teachers are selected as the survey object. They are required to "support," "no objection," and "oppose" the change of the evaluation system by displaying the previous evaluation results and the evaluation results given by the new system; the statistics of three evaluation results are shown in Table 3:

In Table 3, although the evaluation of teachers in the new system is more harsh, the support rate of teachers for the new system has increased from $25.0 \%$ to $37.5 \%$ and the opposition rate has decreased from $62.5 \%$ to $41.7 \%$ compared with the previous system. The fundamental reason is that the evaluation criteria for teachers in the new system are more comprehensive and objective, and teachers can perform more actively in their work, the ratio of labor pay to labor return is more significant. Students' evaluation results of the new system are much higher than those of the previous system, in which the support rate has increased from $29.8 \%$ to $79.2 \%$, and the opposition rate has decreased from $58.2 \%$ to $11.8 \%$.

In Figure 7 , through the analysis of the correlation between the new scheme and the support rate of teachers, the evaluation standard of the new scheme is more objective, which improves the enthusiasm of teachers in all aspects and increases the interaction between teachers and students so 
TABLE 2: Impact of new and old systems on teachers' performance evaluation results.

\begin{tabular}{|c|c|c|c|c|c|c|}
\hline \multirow{2}{*}{ Comparison items } & \multicolumn{3}{|c|}{ Advanced teacher } & \multicolumn{3}{|c|}{ Backward teachers } \\
\hline & Total & Difference & Overlap & Total & Difference & Overlap \\
\hline Previous method & $6(25)$ & - & - & $5(21)$ & - & - \\
\hline New method & $4(17)$ & $3(75)$ & $2(50)$ & $8(33)$ & $1(18)$ & $4(50)$ \\
\hline
\end{tabular}

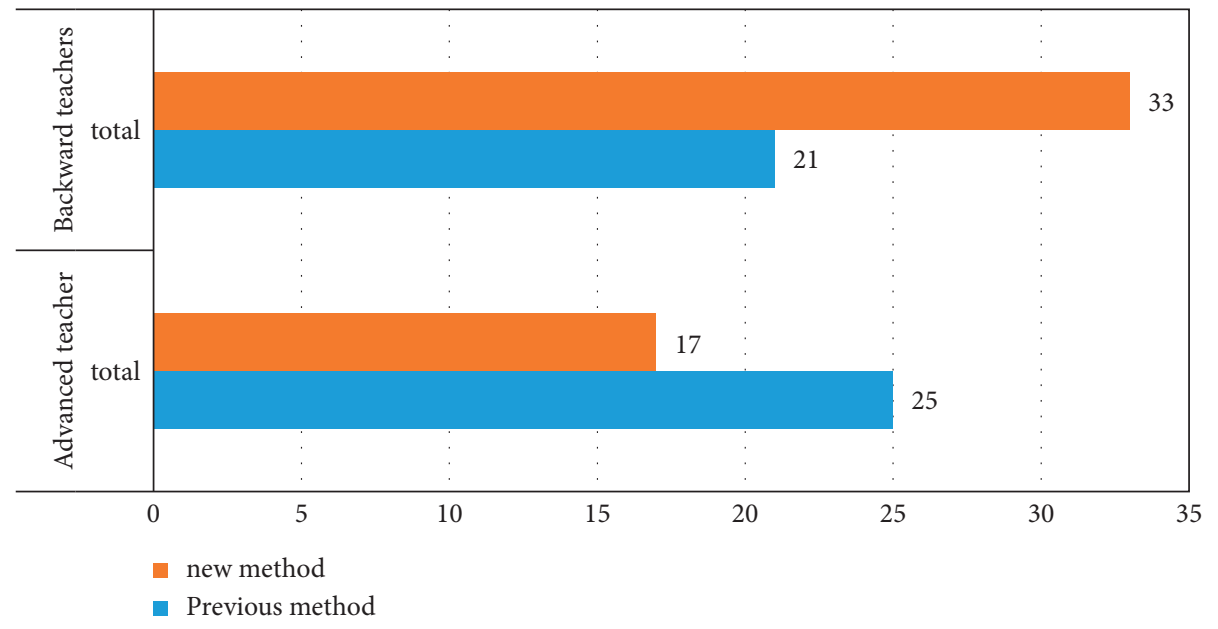

FIGURE 6: Correlation between old and new schemes and teachers' performance evaluation results.

TABle 3: Statistics of support rate for the new system among students and teachers.

\begin{tabular}{|c|c|c|c|c|c|c|}
\hline \multirow{2}{*}{ Comparison items } & \multicolumn{3}{|c|}{ Student $n=500$} & \multicolumn{3}{|c|}{ Teacher $n=24$} \\
\hline & Support & No obj & Oppose & Support & No obj & Oppose \\
\hline Previous method & $149(29.8)$ & $60(12)$ & $291(58.2)$ & $6(25)$ & $3(12.5)$ & $15(62.5)$ \\
\hline New method & $396(79.2)$ & $45(9)$ & $59(11.8)$ & $9(37.5)$ & $5(20.8)$ & $10(41.7)$ \\
\hline
\end{tabular}

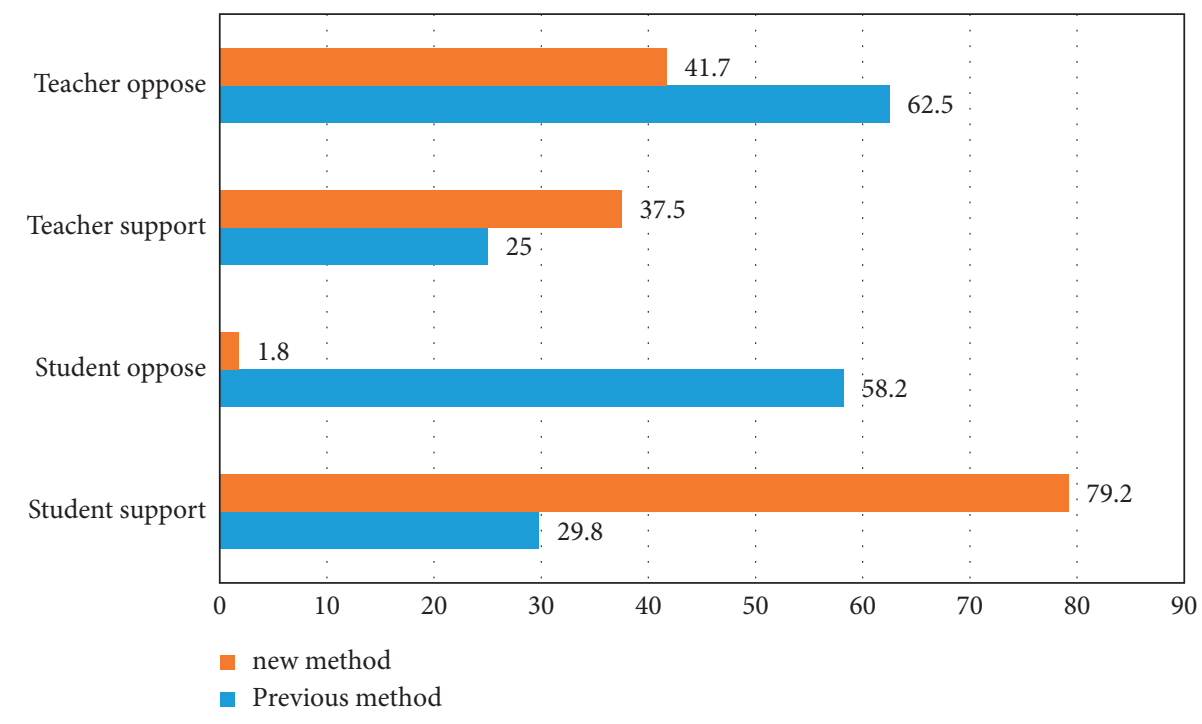

Figure 7: Correlation between the new scheme and the support rate of teachers. 
that the evaluation results of students are much higher than those of the old scheme and the new scheme obtains higher support rate among teachers.

\section{Summary}

In the college physical education management information system, the artificial intelligence evaluation method is used to replace the previous weighted index evaluation method so that the data displayed in the conventional teaching link can intuitively predict the National Physical Education unified examination results, and the evaluation of students' performance and teachers' performance are more objective and comprehensive. However, compared with the comprehensive artificial intelligence system, subject to the historical problems of the current technical system and the college physical education teaching information management system, as well as the linkage restriction of the evaluation mechanism of the surrounding teaching groups, the system cannot realize the comprehensive intervention of the artificial intelligence system in the college physical education teaching process. The follow-up research will further sort out the process, deepen the teaching reform, and further the application of artificial intelligence system.

\section{Data Availability}

No data were used to support this study.

\section{Disclosure}

The authors confirm that the content of the manuscript has not been published or submitted for publication elsewhere.

\section{Conflicts of Interest}

The authors declare that they have no potential conflicts of interest in this study.

\section{Authors' Contributions}

All authors have seen the manuscript and approved to submit to the journal.

\section{References}

[1] Y. Wang and S.-B. Tsai, "Physical education teaching in colleges and universities assisted by virtual reality technology based on artificial intelligence," Mathematical Problems in Engineering, vol. 2021, Article ID 5582716, 11 pages, 2021.

[2] M. Wang, "Design of college physical education teaching system based on artificial intelligence technology," Journal of Physics: Conference Series, vol. 1852, no. 4, 2021.

[3] F. Li, "Information teaching platform of college physical education based on artificial intelligence technology," Journal of Physics: Conference Series, vol. 1852, no. 2, 2021.

[4] J. Wen, "Innovative application of artificial intelligence technology in college physical education," Journal of Physics: Conference Series, vol. 1881, no. 4, 2021.
[5] Z. Li, "Application of artificial intelligence technology in college physical education," Journal of Physics: Conference Series, vol. 1651, no. 1, 2020.

[6] Y. R. Liu, "An artificial intelligence and machine vision based evaluation of physical education teaching," Journal of Intelligent and Fuzzy Systems, vol. 40, no. 2, 2021.

[7] J. Zhang, "Reform and innovation of artificial intelligence technology for information service in university physical education," Journal of Intelligent and Fuzzy Systems, vol. 40, no. 2,2021

[8] Z. Li and H. Wang, "The effectiveness of physical education teaching in college based on Artificial intelligence methods," Journal of Intelligent and Fuzzy Systems, vol. 40, no. 2, 2021.

[9] X. Li and R. Li, "Applying artificial intelligence in physical education and future perspectives," Sustainability, vol. 13, no. $1,2021$.

[10] Gaobin, H. N. Cao and Z. Z. Liu, An artificial intelligence fuzzy system for improvement of physical education teaching method," Journal of Intelligent and Fuzzy Systems, vol. 40, no. 2, 2021.

[11] D. Yang, E. S. Oh, and Y. Wang, "Hybrid physical education teaching and curriculum design based on a voice interactive artificial intelligence educational robot," Sustainability, vol. 12, no. 19, 2020.

[12] B. Yong, "Design and research of intelligent evaluation system of physical education teaching based on artificial intelligence expert decision system," Journal of Residuals Science \& Technology, vol. 13, no. 5, 2016. 